Алгебра и анализ

Том. 18 (2006), № 4
St. Petersburg Math. J.

Vol. 18 (2007), No. 4, Pages 511-516

S 1061-0022(07)00958-2

Article electronically published on May 25, 2007

\title{
INTERPOLATION OF BESOV SPACES IN THE NONDIAGONAL CASE
}

\author{
I. ASEKRITOVA AND N. KRUGLYAK
}

\begin{abstract}
In the nondiagonal case, interpolation spaces for a collection of Besov spaces are described. The results are consequences of the fact that, whenever the convex hull of points $\left(\bar{s}_{0}, \eta_{0}\right), \ldots,\left(\bar{s}_{n}, \eta_{n}\right) \in \mathbb{R}^{m+1}$ includes a ball of $\mathbb{R}^{m+1}$, we have$$
\left(l_{q_{0}}^{\bar{s}_{0}}\left(\left(X_{0}, X_{1}\right)_{\eta_{0}, p_{0}}\right), \ldots, l_{q_{n}}^{\bar{s}_{n}}\left(\left(X_{0}, X_{1}\right)_{\eta_{n}, p_{n}}\right)\right)_{\bar{\theta}, q}=l_{q}^{\bar{s}_{\bar{\theta}}}\left(\left(X_{0}, X_{1}\right)_{\eta_{\bar{\theta}}, q}\right),
$$$$
\text { where } \bar{\theta}=\left(\theta_{0}, \ldots, \theta_{n}\right) \text { and }\left(s_{\bar{\theta}}, \eta_{\bar{\theta}}\right)=\theta_{0}\left(\bar{s}_{0}, \eta_{0}\right)+\cdots+\theta_{n}\left(\bar{s}_{n}, \eta_{n}\right) \text {. }
$$

\section{$\S 0$. INTRODUCTION}

An important problem in interpolation theory is the study of interpolation properties of Besov and Sobolev spaces, either isotropic or anisotropic. It is well known that, in the diagonal case $\left(\frac{1}{q}=\frac{1-\theta}{q_{0}}+\frac{\theta}{q_{1}}, \frac{1}{p}=\frac{1-\theta}{p_{0}}+\frac{\theta}{p_{1}}\right)$, when applied to a couple of isotropic Besov spaces, the real method yields again a Besov space:

$$
\left(B_{p_{0}, q_{0}}^{s_{0}}, B_{p_{1}, q_{1}}^{s_{1}}\right)_{\theta, q}=B_{p, q}^{s_{\theta}} \quad\left(s_{0} \neq s_{1}, p=q, s_{\theta}=(1-\theta) s_{0}+\theta s_{1}\right)
$$

see [3. However, in the nondiagonal case, $(0.1)$ is true only under substantial restrictions on the parameters and, generally speaking, the space $\left(B_{p_{0}, q_{0}}^{s_{0}}, B_{p_{1}, q_{1}}^{s_{1}}\right)_{\theta, q}$ falls out of the Besov scale. At the same time, switching from couples of Besov spaces to triples, we again arrive at a space within the same scale; see [2].

Difficulties presented by interpolation of Besov spaces are related to those arising in interpolation of vector-valued spaces $l_{q}^{s}\left(L_{p}\right)$ (see $\S 1$ for the definition).

In the diagonal case $\left(\frac{1}{q}=\frac{1-\theta}{q_{0}}+\frac{\theta}{q_{1}}\right)$, we have a remarkable formula (see [3] ):

$$
\left(l_{q_{0}}^{s_{0}}\left(A_{0}\right), l_{q_{1}}^{s_{1}}\left(A_{1}\right)\right)_{\theta, q}=l_{q}^{s_{\theta}}\left(\left(A_{0}, A_{1}\right)_{\theta, q}\right),
$$

where $s_{\theta}=(1-\theta) s_{0}+\theta s_{1}$. But in the nondiagonal case $\left(\frac{1}{q} \neq \frac{1-\theta}{q_{0}}+\frac{\theta}{q_{1}}\right)$ this formula cannot be valid for an arbitrary couple $\left(A_{0}, A_{1}\right)$.

This can be shown by a very simple example. Consider the couple $\left(l_{p}^{s_{0}}\left(A_{0}\right), l_{p}^{s_{1}}\left(A_{1}\right)\right)$, where $s_{0} \neq s_{1}$, and the inner spaces $A_{0}$ and $A_{1}$ are $l_{p}^{r_{0}}$ and $l_{p}^{r_{1}}\left(r_{0} \neq r_{1}\right)$, respectively. Then, by the Gilbert formula [5], the space

$$
X=\left(l_{p}^{s_{0}}\left(l_{p}^{r_{0}}\right), l_{p}^{s_{1}}\left(l_{p}^{r_{1}}\right)\right)_{\theta, q}, \quad q \neq p,
$$

corresponds to the norm

$$
\left\|\left\{a_{n_{1}, n_{2}}\right\}_{\left(n_{1}, n_{2}\right) \in \mathbb{Z}^{2}}\right\|_{X}=\left(\sum_{k}\left\|2^{s_{\theta} n_{1}} 2^{r_{\theta} n_{2}} a_{n_{1}, n_{2}} \chi_{\Omega_{k}}\right\|_{l_{p}}^{q}\right)^{1 / q},
$$

2000 Mathematics Subject Classification. Primary 46B70; Secondary 46E30.

Key words and phrases. Real interpolation, vector-valued spaces, Besov spaces. 
where $\Omega_{k}=\left\{\left(n_{1}, n_{2}\right): 2^{k-1}<2^{n_{1}\left(s_{0}-s_{1}\right)} 2^{n_{2}\left(r_{0}-r_{1}\right)} \leq 2^{k}\right\}, s_{\theta}=(1-\theta) s_{0}+\theta s_{1}$, and $r_{\theta}=(1-\theta) r_{0}+\theta r_{1}$. Clearly, this is not the norm of the space

$$
l_{q}^{s_{\theta}}\left(l_{q}^{r_{\theta}}\right)=l_{q}^{s_{\theta}}\left(\left(A_{0}, A_{1}\right)_{\theta, q}\right)
$$

if $q \neq p$.

Thus, for arbitrary $A_{0}$ and $A_{1}$, formula (0.2) fails in the nondiagonal case. However, as was shown in 1, it can be extended to the nondiagonal case if we consider a collection of more than two vector-valued spaces in place of a couple. Specifically, if $A_{n}=A_{n-1}$, $s_{n} \neq s_{n-1}$, then

$$
\left(l_{q_{0}}^{s_{0}}\left(A_{0}\right), l_{q_{1}}^{s_{1}}\left(A_{1}\right), \ldots, l_{q_{n}}^{s_{n}}\left(A_{n}\right)\right)_{\bar{\theta}, q}=l_{q}^{s_{\bar{\theta}}}\left(\left(A_{0}, \ldots, A_{n}\right)_{\bar{\theta}, q}\right),
$$

where $\bar{\theta}=\left(\theta_{0}, \theta_{1}, \ldots, \theta_{n}\right), s_{\bar{\theta}}=\sum_{i=0}^{n} \theta_{i} s_{i}$. It is worthy of notice that there are no restrictions on $A_{0}, A_{1}, \ldots, A_{n-2}$ and $s_{0}, s_{1}, \ldots, s_{n-2}$ in $(0.3)$.

Let $\bar{s}_{k} \in \mathbb{R}^{m}, k=0,1, \ldots, n$, let $\vec{X}=\left(X_{0}, X_{1}\right)$ be a couple of Banach or quasi-Banach spaces, and let $A_{k}=\left(X_{0}, X_{1}\right)_{\lambda_{k}, p_{k}}, k=0,1, \ldots, n$. We shall show that formula $(0.3)$ extends to the case of the collection

$$
\left(l_{q_{0}}^{\bar{s}_{0}}\left(A_{0}\right), l_{q_{1}}^{\bar{s}_{1}}\left(A_{1}\right), \ldots, l_{q_{n}}^{\bar{s}_{n}}\left(A_{n}\right)\right)
$$

as a consequence, we shall be able to interpolate collections of Besov and Sobolev spaces in the nondiagonal case.

\section{$\S 1$. BASIC DEFINITIONS}

As usual, we denote by $\mathbb{R}_{+}^{n}$ the set of $n$-vectors with positive coordinates.

Let $A_{0}, A_{1}, \ldots, A_{n}$ be Banach or quasi-Banach spaces. We say that $A_{0}, A_{1}, \ldots, A_{n}$ constitute an $(n+1)$-tuple if they are embedded linearly and continuously in a certain Hausdorff linear topological space $X$.

Much as for the case of couples, we can define the $K$-functional of an element $a \in$ $A_{0}+A_{1}+\cdots+A_{n}$ :

$$
K(\bar{t}, a, \vec{A})=\inf \left(\left\|a_{0}\right\|_{A_{0}}+t_{1}\left\|a_{1}\right\|_{A_{1}}+\cdots+t_{n}\left\|a_{n}\right\|_{A_{n}}\right),
$$

where $\bar{t}=\left(t_{1}, \ldots, t_{n}\right) \in \mathbb{R}_{+}^{n}$, and the infimum is taken over all representations $a=$ $a_{0}+a_{1}+\cdots+a_{n}$ with $a_{i} \in A_{i}, i=0,1, \ldots, n$.

Clearly, the $K$-functional is a concave function on $\mathbb{R}_{+}^{n}$; for fixed $t$, this is a norm on $A_{0}+\cdots+A_{n}$.

Next, for every parameter $\bar{\theta}=\left(\theta_{0}, \ldots, \theta_{n}\right), \theta_{i}>0, i=0,1, \ldots, n$, with $\sum_{i=0}^{n} \theta_{i}=1$, we define the interpolation space $\vec{A}_{\bar{\theta}, q}, q>0$, as the collection of all elements $a \in$ $A_{0}+A_{1}+\cdots+A_{n}$ for which the quasinorm

$$
\|a\|_{\bar{\theta}, q}=\left(\int_{\mathbb{R}_{+}^{n}}\left(t_{1}^{-\theta_{1}} \cdots t_{n}^{-\theta_{n}} K(\bar{t}, a ; \bar{A})\right)^{q} \frac{d t_{1}}{t_{1}} \cdots \frac{d t_{n}}{t_{n}}\right)^{1 / q}
$$

is finite (for $q \geq 1$ this is a norm). As usual, the integral is replaced with the supremum if $q=\infty$.

Let $A$ be a Banach or quasi-Banach space. Suppose $\bar{s}=\left(s_{1}, \ldots, s_{m}\right) \in \mathbb{R}^{m}, q>0$, and $\bar{k}=\left(k_{1}, \ldots, k_{m}\right) \in \mathbb{Z}^{m}$. We denote by $l_{q}^{\bar{s}}(A)$ the space of $A$-valued functions determined by the following norm (quasinorm if $0<q<1$ ):

$$
\|a\|_{l_{q}^{\bar{s}}(A)}=\left\|\left\{a_{k}\right\}_{k \in \mathbb{Z}^{m}}\right\|_{l_{q}^{\bar{s}}(A)}=\left(\sum_{\bar{k} \in \mathbb{Z}^{m}}\left(2^{\bar{k} \cdot \bar{s}}\left\|a_{\bar{k}}\right\|_{A}\right)^{q}\right)^{1 / q} ;
$$

here $\bar{k} \cdot \bar{s}=\sum_{i=1}^{m} k_{i} s_{i}$, and the sum in (1.3) is replaced with the supremum if $q=\infty$. 


\section{§2. INTERPOLATION OF SPACES OF VECTOR-VALUED FUNCTIONS}

Suppose $\bar{s}_{k}=\left(s_{1}^{k}, \ldots, s_{m}^{k}\right) \in \mathbb{R}^{m}, \eta_{k} \in(0,1), k=0,1, \ldots, n$. We denote by $\left(\bar{s}_{k}, \eta_{k}\right)$ the point of $\mathbb{R}^{m+1}$ with the coordinates $\left(s_{1}^{k}, \ldots, s_{m}^{k}, \eta_{k}\right)$.

Let $\vec{X}=\left(X_{0}, X_{1}\right)$ be a couple of Banach or quasi-Banach spaces.

Theorem 1. If the convex hull of the $(n+1)$-tuple $\left(\bar{s}_{0}, \eta_{0}\right),\left(\bar{s}_{1}, \eta_{1}\right), \ldots,\left(\bar{s}_{n}, \eta_{n}\right)$ contains some ball of $\mathbb{R}^{m+1}$, then

$$
\left(l_{q_{0}}^{\bar{s}_{0}}\left(\vec{X}_{\eta_{0}, p_{0}}\right), \ldots, l_{q_{n}}^{\bar{s}_{n}}\left(\vec{X}_{\eta_{n}, p_{n}}\right)\right)_{\bar{\theta}, q}=l_{q}^{\bar{s}_{\bar{\theta}}}\left(\vec{X}_{\eta_{\bar{\theta}}, q}\right),
$$

where $\left(\bar{s}_{\bar{\theta}}, \eta_{\bar{\theta}}\right)=\theta_{0}\left(\bar{s}_{0}, \eta_{0}\right)+\cdots+\theta_{n}\left(\bar{s}_{n}, \eta_{n}\right)$.

For the proof, we need the following lemma.

Lemma 1. Let $\bar{e}_{i}(i=1, \ldots, m)$ be the standard basis of $\mathbb{R}^{m}$. Put $\bar{e}_{0}=\bar{e}_{m+1}=$ $(0, \ldots, 0) \in \mathbb{R}^{m}$. Then for every $a \neq 0$ we have

$$
\left(l_{p}^{a \bar{e}_{0}}\left(X_{0}\right), l_{p}^{a \bar{e}_{1}}\left(X_{1}\right), \ldots, l_{p}^{a \bar{e}_{m+1}}\left(X_{1}\right)\right)_{\bar{\theta}, q}=l_{q}^{a\left(\theta_{1}, \ldots, \theta_{m}\right)}\left(\vec{X}_{\theta_{1}+\cdots+\theta_{m+1}, q}\right),
$$

where $\bar{\theta}=\left(\theta_{0}, \theta_{1}, \ldots, \theta_{m+1}\right)$.

Proof. We proceed by induction on $m$. If $m=1$, then $\bar{e}_{0}=\bar{e}_{2}=0, a \bar{e}_{1}=a \neq 0$. Therefore, formula (2.2) takes the form

$$
\left(l_{p}\left(X_{0}\right), l_{p}^{a}\left(X_{1}\right), l_{p}\left(X_{1}\right)\right)_{\bar{\theta}, q}=l_{q}^{a \theta_{1}}\left(\vec{X}_{\theta_{1}+\theta_{2}, q}\right),
$$

where $\bar{\theta}=\left(\theta_{0}, \theta_{1}, \theta_{2}\right)$. This is true indeed by $(0.3)$ with $n=2$.

Suppose (2.2) is true for $m \leq N$. We show it is true for $m=N+1$; namely, we prove the formula

$$
\left(l_{p}^{a \bar{e}_{0}}\left(X_{0}\right), l_{p}^{a \bar{e}_{1}}\left(X_{1}\right), \ldots, l_{p}^{a \bar{e}_{N+2}}\left(X_{1}\right)\right)_{\bar{\theta}, q}=l_{q}^{a\left(\theta_{1}, \ldots, \theta_{N+1}\right)}\left(\vec{X}_{\theta_{1}+\cdots+\theta_{N+2}, q}\right),
$$

where $\bar{\theta}=\left(\theta_{0}, \ldots, \theta_{N+1}, \theta_{N+2}\right)$.

We denote by $\bar{e}_{i}^{N}, i=0,1, \ldots, N+2$, the vectors in $\mathbb{R}^{N}$ with coordinates equal to the first $N$ coordinates of the vectors $\bar{e}_{i}$, and consider the spaces

$$
Y_{0}=l_{p}^{a \bar{e}_{0}^{N}}\left(X_{0}\right), \quad Y_{i}=l_{p}^{a \bar{e}_{i}^{N}}\left(X_{1}\right), \quad i=1, \ldots, N+2 .
$$

It is easily seen that the space on the left in (2.4) can be rewritten in the form

$$
\begin{aligned}
& \left(l_{p}^{a \bar{e}_{0}}\left(X_{0}\right), l_{p}^{a \bar{e}_{1}}\left(X_{1}\right), \ldots, l_{p}^{a \bar{e}_{N+2}}\left(X_{1}\right)\right)_{\bar{\theta}, q} \\
& \quad=\left(l_{p}\left(Y_{0}\right), l_{p}\left(Y_{1}\right), \ldots, l_{p}^{a}\left(Y_{N+1}\right), l_{p}\left(Y_{N+2}\right)\right)_{\bar{\theta}, q} .
\end{aligned}
$$

Since $\bar{e}_{N+1}^{N}=\bar{e}_{N+2}^{N}=\overline{0} \in \mathbb{R}^{N}$ and $a \neq 0$, we see that $Y_{N+1}=Y_{N+2}$, and $(0.3)$ can be applied to the right-hand side of (2.6). This yields

$$
\begin{aligned}
\left(l_{p}^{a \bar{e}_{0}}\right. & \left.\left(X_{0}\right), l_{p}^{a \bar{e}_{1}}\left(X_{1}\right), \ldots, l_{p}^{a \bar{e}_{N+2}}\left(X_{1}\right)\right)_{\bar{\theta}, q} \\
& =l_{q}^{a \theta_{N+1}}\left(\left(Y_{0}, \ldots, Y_{N+1}, Y_{N+2}\right)_{\bar{\theta}, q}\right) \\
& =l_{q}^{a \theta_{N+1}}\left(\left(Y_{0}, \ldots, Y_{N+1}\right)_{\theta_{0}, \ldots, \theta_{N+1}+\theta_{N+2}, q}\right) .
\end{aligned}
$$

It remains to observe that the collection $\left(Y_{0}, \ldots, Y_{N+1}\right)$ satisfies the inductive hypothesis. Therefore,

$$
\begin{aligned}
\left(l_{p}^{a \bar{e}_{0}}\right. & \left.\left(X_{0}\right), l_{p}^{a \bar{e}_{1}}\left(X_{1}\right), \ldots, l_{p}^{a \bar{e}_{N+2}}\left(X_{1}\right)\right)_{\bar{\theta}, q} \\
& =l_{q}^{a \theta_{N+1}}\left(l_{q}^{a\left(\theta_{1}, \ldots, \theta_{N}\right)}\left(\left(X_{0}, X_{1}\right)_{\theta_{1}+\cdots+\theta_{N+2}, q}\right)\right) \\
& =l_{q}^{a\left(\theta_{1}, \ldots, \theta_{N+1}\right)}\left(\vec{X}_{\theta_{1}+\cdots+\theta_{N+2}, q}\right) .
\end{aligned}
$$

The lemma is proved. 
Proof of Theorem 1. The right-hand side of (2.1) does not depend on the $p_{i}$ and $q_{i}(i=$ $0,1, \ldots, n)$. Therefore, the continuous embeddings

$$
l_{r_{0}}^{\bar{s}_{i}}\left(\vec{X}_{\eta_{i}, r_{0}}\right) \hookrightarrow l_{q_{i}}^{\bar{s}_{i}}\left(\vec{X}_{\eta_{i}, p_{i}}\right) \hookrightarrow l_{r_{1}}^{\bar{s}_{i}}\left(\vec{X}_{\eta_{i}, r_{1}}\right)
$$

where

$$
r_{0}=\min _{0 \leq i, j \leq n}\left\{p_{i}, q_{j}\right\}, \quad r_{1}=\max _{0 \leq i, j \leq n}\left\{p_{i}, q_{j}\right\},
$$

show that it suffices to prove Theorem 1 only in the case where all $p_{i}$ and $q_{i}, i=0, \ldots, n$, are equal to one and the same number $r$. Namely, it suffices to prove that

$$
\left(l_{r}^{\bar{s}_{0}}\left(\vec{X}_{\eta_{0}, r}\right), \ldots, l_{r}^{\bar{s}_{n}}\left(\vec{X}_{\eta_{n}, r}\right)\right)_{\bar{\theta}, q}=l_{q}^{\bar{s}_{\theta}}\left(\vec{X}_{\eta_{\bar{\theta}}, q}\right) .
$$

Next, without loss of generality we may assume that $\bar{s}_{i} \in \mathbb{R}_{+}^{m}, i=0,1, \ldots, n$. Indeed, since

$$
\begin{aligned}
& \left\|\left\{a_{\bar{k}}\right\}_{\bar{k} \in \mathbb{Z}^{m}}\right\|_{l_{r}^{\bar{s}_{i}+\bar{s}}\left(\vec{X}_{\eta_{i}, r}\right)} \\
& \quad=\left(\sum_{k \in \mathbb{Z}^{m}}\left(2^{\left(\bar{s}_{i}+\bar{s}\right) \cdot \bar{k}}\left\|a_{\bar{k}}\right\|_{\bar{X}_{\eta_{i}, r}}\right)^{r}\right)^{1 / r}=\left\|\left\{2^{\bar{s} \cdot \bar{k}} a_{\bar{k}}\right\}_{\bar{k} \in \mathbb{Z}^{m}}\right\|_{l_{r}^{\bar{s}_{i}}\left(\vec{X}_{\eta_{i}, r}\right)},
\end{aligned}
$$

the mapping

$$
A_{\bar{s}}:\left\{a_{\bar{k}}\right\}_{\bar{k} \in \mathbb{Z}^{m}} \mapsto\left\{2^{\bar{s} \cdot \bar{k}} a_{\bar{k}}\right\}_{\bar{k} \in \mathbb{Z}^{m}}
$$

is an isometry between $l_{r}^{\bar{s}_{i}+\bar{s}}\left(\vec{X}_{\eta_{i}, r}\right)$ and $l_{r}^{\bar{s}_{i}}\left(\vec{X}_{\eta_{i}, r}\right)$ and also between $l_{q}^{\bar{s}_{\bar{\theta}}+\bar{s}}\left(\vec{X}_{\eta_{\bar{\theta}}, q}\right)$ and $l_{q}^{\bar{s}_{\bar{\theta}}}\left(\vec{X}_{\eta_{\bar{\theta}}, q}\right)$. Moreover,

$$
\theta_{0}\left(\bar{s}_{0}+\bar{s}\right)+\theta_{1}\left(\bar{s}_{1}+\bar{s}\right)+\cdots+\theta_{n}\left(\bar{s}_{n}+\bar{s}\right)=\bar{s}_{\bar{\theta}}+\bar{s} .
$$

Consequently, it suffices to prove (2.9) for $\bar{s}_{i} \in \mathbb{R}_{+}^{m}, i=0, \ldots, n$.

Now, we show that if $\bar{s}_{i} \in \mathbb{R}_{+}^{m}$, then all spaces $l_{r}^{\bar{s}_{i}}\left(\vec{X}_{\eta_{i}, r}\right)$ can be obtained by the real method of interpolation from the "basic" collection

$$
\vec{Z}=\left(l_{r}^{a \bar{e}_{0}}\left(X_{0}\right), l_{r}^{a \bar{e}_{1}}\left(X_{1}\right), \ldots, l_{r}^{a \bar{e}_{m+1}}\left(X_{1}\right)\right),
$$

treated in Lemma 1.

For this, we choose a sufficiently large number $a\left(a>\max _{i=0, n} \frac{\sum_{j=1}^{m} s_{j}^{i}}{\eta_{i}}\right)$ and define vectors $\bar{\lambda}_{i}=\left(\lambda_{0}^{i}, \ldots, \lambda_{m+1}^{i}\right), i=0,1, \ldots, n$, in the following way:

$$
\lambda_{j}^{i}=\frac{1}{a} s_{j}^{i}, \quad j=1, \ldots, m, \quad \lambda_{m+1}^{i}=\eta_{i}-\sum_{j=1}^{m} \frac{s_{j}^{i}}{a} .
$$

Also, we put $\lambda_{0}^{i}=1-\sum_{j=1}^{m+1} \lambda_{j}^{i}$. Clearly, if $a$ is sufficiently large, then all $\lambda_{j}^{i}(j=$ $0, \ldots, m+1)$ are strictly positive and $\sum_{j=0}^{m+1} \lambda_{j}^{i}=1$. Therefore, from Lemma 1 and formula (2.12) we deduce that

$$
\left(l_{r}^{a \bar{e}_{0}}\left(X_{0}\right), l_{r}^{a \bar{e}_{1}}\left(X_{1}\right), \ldots, l_{r}^{a \bar{e}_{m+1}}\left(X_{1}\right)\right)_{\bar{\lambda}_{i}, r}=l_{r}^{a\left(\lambda_{1}^{i}, \ldots, \lambda_{m}^{i}\right)}\left(\vec{X}_{\lambda_{1}^{i}+\cdots+\lambda_{m+1}^{i}, r}\right)=l_{r}^{\bar{s}_{i}}\left(\vec{X}_{\eta_{i}, r}\right)
$$

for all $i=0,1, \ldots, n$.

Thus, the left-hand side of (2.9) has the form

$$
\left(l_{r}^{\bar{s}_{0}}\left(\vec{X}_{\eta_{0}, r}\right), l_{r}^{\bar{s}_{1}}\left(\vec{X}_{\eta_{1}, r}\right), \ldots, l_{r}^{\bar{s}_{n}}\left(\vec{X}_{\eta_{n}, r}\right)\right)_{\bar{\theta}_{, q}}=\left(\bar{Z}_{\bar{\lambda}_{0}, r}, \bar{Z}_{\bar{\lambda}_{1}, r}, \ldots, \bar{Z}_{\bar{\lambda}_{n}, r}\right)_{\bar{\theta}, q},
$$

where $\vec{Z}$ is defined by (2.11). Next, from [6, Propositions 6.4, 10.1, and Theorem 1], the reiteration theorem applies to the collection $\bar{Z}$ :

$$
\left(\bar{Z}_{\bar{\lambda}_{0}, r}, \bar{Z}_{\bar{\lambda}_{1}, r}, \ldots, \bar{Z}_{\bar{\lambda}_{n}, r}\right)_{\bar{\theta}, q}=\bar{Z}_{\theta_{0} \bar{\lambda}_{0}+\cdots+\theta_{n} \bar{\lambda}_{n}, q}
$$

provided that the linear hull of the vectors $\bar{\lambda}_{0}, \bar{\lambda}_{1}, \ldots, \bar{\lambda}_{n}$ coincides with $\mathbb{R}^{m+2}$. Since the convex hull of the $\left(\bar{s}_{i}, \eta_{i}\right) \in \mathbb{R}^{m+1}, i=0,1, \ldots, n$, includes some ball of $\mathbb{R}^{m+1}$, and 
the points $p_{i}=\left(\lambda_{1}^{i}, \lambda_{2}^{i}, \ldots, \lambda_{m+1}^{i}\right), i=0,1, \ldots, n$, are obtained from the $\left(\bar{s}_{i}, \eta_{i}\right)$ with the help of a nondegenerate linear mapping (see (2.12)), we see that the convex hull of the $p_{i}$ also includes some ball of $\mathbb{R}^{m+1}$. Consequently, the convex hull of the points $\left(\lambda_{0}^{i}, \lambda_{1}^{i}, \ldots, \lambda_{m+1}^{i}\right) \in \mathbb{R}^{m+2}, i=0,1, \ldots, n$, which lie on the hyperplane $t_{0}+t_{1}+\cdots+$ $t_{m+1}=1$, also includes a ball of dimension $m+1$. This implies immediately that the linear hull of the vectors $\bar{\lambda}_{0}, \bar{\lambda}_{1}, \ldots, \bar{\lambda}_{n}$ is the entire $\mathbb{R}^{m+2}$.

Now, from formulas (2.14) and (2.15), Lemma 1, and identity (2.12) we obtain

$$
\begin{aligned}
& \left(l_{r}^{\bar{s}_{0}}\left(\vec{X}_{\eta_{0}, r}\right), l_{r}^{\bar{s}_{1}}\left(\vec{X}_{\eta_{1}, r}\right), \ldots, l_{r}^{\bar{s}_{n}}\left(\vec{X}_{\eta_{n}, r}\right)\right)_{\bar{\theta}, q} \\
& \quad=\left(l_{r}^{a \bar{e}_{0}}\left(X_{0}\right), l_{r}^{a \bar{e}_{1}}\left(X_{1}\right), \ldots, l_{r}^{a \bar{e}_{m+1}}\left(X_{1}\right)\right)_{\bar{\lambda}_{\bar{\theta}}, q}=l_{q}^{\bar{s}_{\theta}}\left(\vec{X}_{\bar{\eta}_{\bar{\theta}}}, q\right),
\end{aligned}
$$

which completes the proof of the theorem.

Theorem 1 treats the collection of spaces

$$
l_{q_{i}}^{\bar{s}_{i}}\left(\vec{X}_{\eta_{i}, p_{i}}\right)=l_{q_{i}}^{\bar{s}_{i}}\left(\left(X_{0}, X_{1}\right)_{\eta_{i}, p_{i}}\right)
$$

here, as usual, $0<\eta_{i}<1, i=0, \ldots, n$. However, the case where the "extreme" space $X_{1}$ is involved in place of some spaces $\left(X_{0}, X_{1}\right)_{\eta_{i}, p_{i}}$ is of interest. Then we put $X_{1}=\left(X_{0}, X_{1}\right)_{1, p_{i}}$, i.e., the parameter $\eta_{i}$ may belong to the semi-interval $(0,1]$.

Remark. Theorem 1 remains true in the case where $\eta_{i} \in(0,1]$.

This follows immediately from the fact that Lemma 1 is true also for $\theta_{0}=0$ and the reiteration theorem (see [6]) is true also in the case where some coordinates of $\bar{\theta}$ are equal to 0 .

\section{§3. Applichtions}

Consider the collection of vector-valued spaces

$$
\left(l_{q_{0}}^{\bar{s}_{0}}\left(L_{p_{0}}\right), \ldots, l_{q_{n}}^{\bar{s}_{n}}\left(L_{p_{n}}\right)\right),
$$

where $\bar{s}_{i} \in \mathbb{R}^{m}, m \geq 1, p_{i}, q_{i}>0, i=0,1, \ldots, n$.

Since for $r<\min _{0 \leq i \leq n} p_{i}$ we have

$$
L_{p_{i}}=\left(L_{r}, L_{\infty}\right)_{\eta_{i}, p_{i}}, \quad \eta_{i}=1-\frac{r}{p_{i}}, \quad i=0, \ldots, n,
$$

Theorem 1 readily implies the following statement.

Theorem 2. If vectors $\bar{s}_{0}, \ldots, \bar{s}_{n} \in \mathbb{R}^{m}$ have the property that the convex hull of the vectors $\left(\bar{s}_{0}, \frac{1}{p_{0}}\right), \ldots,\left(\bar{s}_{n}, \frac{1}{p_{n}}\right)$ includes a ball of $\mathbb{R}^{m+1}$, then

$$
\left(l_{q_{0}}^{\bar{s}_{0}}\left(L_{p_{0}}\right), \ldots, l_{q_{n}}^{\bar{s}_{n}}\left(L_{p_{n}}\right)\right)_{\bar{\theta}, q}=l_{q}^{\bar{s}_{\theta}}\left(L_{p_{\theta}, q}\right),
$$

where $L_{p_{\theta}, q}$ is the Lorentz space and $\left(\bar{s}_{\bar{\theta}}, \frac{1}{p_{\theta}}\right)=\theta_{0}\left(\bar{s}_{0}, \frac{1}{p_{0}}\right)+\cdots+\theta_{n}\left(\bar{s}_{n}, \frac{1}{p_{n}}\right)$.

Theorem 2 and the fact that Besov spaces are retracts of $l_{q}^{\bar{s}}\left(L_{p}\right)$ imply interpolation formulas for Besov and Sobolev spaces.

We start with the isotropic case. It is known (see [3] for $q, p \geq 1$ and [4] for $q, p<1$ ) that $B_{q}^{s}\left(L_{p}\right)$ (i.e., the Besov spaces of smoothness $s$ constructed on the basis of $L_{p}$ ) are obtained from $l_{q}^{s}\left(L_{p}\right)$ with the help of some retraction independent of $p, q$, and $s$. Therefore, Theorem 2 implies the following statement.

Corollary 1. If the convex hull of the points $\left(s_{0}, \frac{1}{p_{0}}\right), \ldots,\left(s_{n}, \frac{1}{p_{n}}\right)$ includes a ball of $\mathbb{R}^{2}$ (i.e., the points do not lie on one straight line), then

$$
\left(B_{q_{0}}^{s_{0}}\left(L_{p_{0}}\right), \ldots, B_{q_{n}}^{s_{n}}\left(L_{p_{n}}\right)\right)_{\bar{\theta}, q}=B_{q}^{s_{\bar{\theta}}}\left(L_{p_{\bar{\theta}}, q}\right),
$$

where $\left(s_{\bar{\theta}}, \frac{1}{p_{\bar{\theta}}}\right)=\theta_{0}\left(s_{0}, \frac{1}{p_{0}}\right)+\cdots+\theta_{n}\left(s_{n}, \frac{1}{p_{n}}\right)$. 
Since for Sobolev spaces we have

$$
B_{1}^{s}\left(L_{p}\right) \subset W_{p}^{s} \subset B_{\infty}^{s}\left(L_{p}\right),
$$

Corollary 1 implies yet another statement.

Corollary 2. Under the assumptions of Corollary 1, we have

$$
\left(W_{p_{0}}^{s_{0}}, \ldots, W_{p_{n}}^{s_{n}}\right)_{\bar{\theta}, q}=B_{q}^{s_{\bar{\theta}}}\left(L_{p_{\bar{\theta}}, q}\right),
$$

where $\left(s_{\bar{\theta}}, \frac{1}{p_{\theta}}\right)=\theta_{0}\left(s_{0}, \frac{1}{p_{0}}\right)+\cdots+\theta_{n}\left(s_{n}, \frac{1}{p_{n}}\right)$.

Similar results are valid also for anisotropic Besov and Sobolev spaces with dominating smoothness.

It is well known (see, e.g., [6]) that a Besov space with dominating smoothness $\bar{s} \in \mathbb{R}^{m}$ constructed on the basis of $L_{p}, p \geq 1$ (it will be denoted by $\tilde{B}_{q}^{\bar{s}}\left(L_{p}\right)$ ) is a retract of $l_{q}^{\bar{s}}\left(L_{p}\right)$. We denote by $\tilde{B}_{q}^{\bar{s}}\left(L_{p, r}\right)$ the space obtained from $l_{q}^{\bar{s}}\left(L_{p, r}\right)$ under the same retraction. Then Theorem 2 implies immediately the following statement.

Corollary 3. If the convex hull of the points $\left(\bar{s}_{0}, \frac{1}{p_{0}}\right), \ldots,\left(\bar{s}_{n}, \frac{1}{p_{n}}\right)$ contains a ball of $\mathbb{R}^{m+1}$, then

$$
\left(\tilde{B}^{\bar{s}_{0}}\left(L_{p_{0}}\right), \ldots, \tilde{B}^{\bar{s}_{n}}\left(L_{p_{n}}\right)\right)_{\bar{\theta}, q}=\tilde{B}_{q}^{\bar{s}_{\bar{\theta}}}\left(L_{p_{\bar{\theta}}, q}\right),
$$

where $\bar{s}_{\bar{\theta}}$ and $p_{\bar{\theta}}$ are defined by

$$
\left(\bar{s}_{\bar{\theta}}\right)=\theta_{0}\left(\bar{s}_{0}, \frac{1}{p_{0}}\right)+\cdots+\theta_{n}\left(\bar{s}_{n}, \frac{1}{p_{n}}\right) .
$$

\section{REFERENCES}

[1] I. Asekritova and N. Krugljak, Real interpolation of vector-valued spaces in non-diagonal case, Proc. Amer. Math. Soc. 133 (2005), 1665-1675. MR2120255 (2005k:46047)

[2] I. Asekritova, N. Krugljak, L. Maligranda, L. Nikolova, and L.-E. Persson, Lions-Peetre reiteration formulas for triples and their applications, Studia Math. 145 (2001), 219-254. MR1829209 (2002e:46089)

[3] J. Bergh and J. Löfström, Interpolation spaces. An introduction, Grundlehren Math. Wiss., vol. 223, Springer-Verlag, Berlin-New York, 1976. MR0482275 (58:2349)

[4] R. DeVore and V. Popov, Interpolation of approximation spaces, Constructive Theory of Functions (Varna, 1987), Bulgar. Acad. Sci., Sofia, 1988, pp. 110-119. MR994826 (90e:46076)

[5] J. E. Gilbert, Interpolation between weighted $L^{p}$-spaces, Ark. Mat. 10 (1972), 235-249. MR0324393 $(48: 2745)$

[6] G. Sparr, Interpolation of several Banach spaces, Ann. Mat. Pura Appl. (4) 99 (1974), 247-316. MR0372641 (51:8848)

School of Mathematics and System Engineering, Växjö University, Sweden

E-mail address: irina.asekritova@vxu.se

Department of Mathematics, Lulea University of Technology, Sweden

E-mail address: natan@ltu.se

Received 21/JAN/2006

Translated by S. V. KISLYAKOV 